\title{
Table of legislation
}

\section{China \\ Anti-Unfair Competition Law (AUCL), 1993 206, 207 Art. 10 .205 \\ Civil Procedure Law Art. 100 \\ Company Law \\ Contract Law \\ Copyright Law 2010 Art. 11 \\ Criminal Law \\ General Principles of the Civil Law \\ Labor Law .206 204 204 233 204 \\ Labor Contract Law 2008 ...73, 204, 283 Regulations on Technology Import and Export Administration} Art. 27

Art. 29 $.264,265$ .264

\section{France}

Code of Industrial Property. 202

Copyright Code

Art. L. 111-1

Art. L. 111-1(2)

Art. L. 113-9

Art. L. 121-1

Art. L. 121-2

Art. L. 121-4

Art. L. 121-7

Art. L. 122-6

Art. L. 131-1

Art. L. 131-3

Intellectual Property Code 239,240

240 240,241

.240

238,239

239

241

238

240

240

240

\section{Germany}

Copyright Act

Art. 1

Art. 2(1)

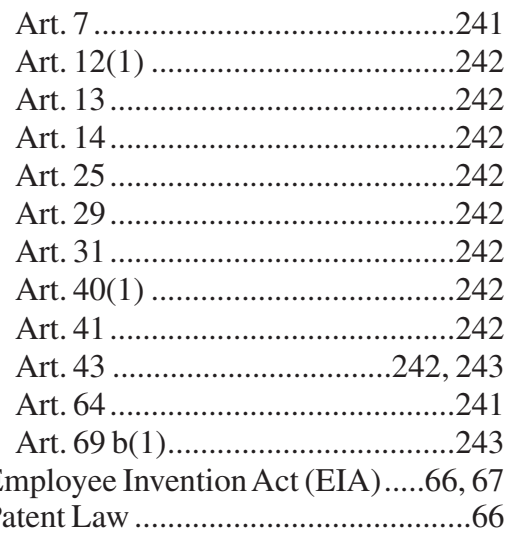

\section{India}

Contract Act 1872 ..........................212

Copyright Act 1957 .........................245

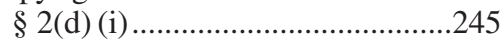

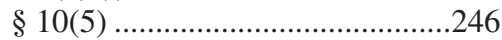

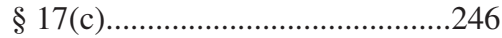

$\S 18(1) \ldots \ldots \ldots \ldots \ldots \ldots \ldots \ldots \ldots \ldots \ldots \ldots \ldots . . .246$

$\S 19(1) \ldots \ldots \ldots \ldots \ldots \ldots \ldots \ldots \ldots \ldots \ldots \ldots \ldots . . .246$

§ 19(4) ......................................246

§ 52(1) ........................................247

§ 52(1)(aa) .................................247

$\S 57(1) \ldots \ldots \ldots \ldots \ldots \ldots \ldots \ldots \ldots \ldots \ldots \ldots . . .246$

$\S \S 57(1)(a),(b) \ldots \ldots \ldots \ldots \ldots \ldots \ldots \ldots \ldots . . .246$

Patent Act 1970

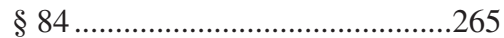

§ 84(1) ..........................................265

$\S 140 \ldots \ldots \ldots \ldots \ldots \ldots \ldots \ldots \ldots \ldots \ldots \ldots \ldots . . . . .265$

Penal Code 1860

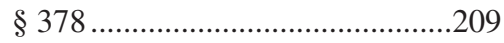

\section{Japan}

Patent Act, Act No. 121 of 1959

Art. 35 .66 


\section{United Kingdom}

Copyright, Designs and Patent Act 1988 .239

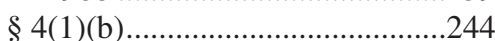

$\S 8$ .245

§ 9 243

$\S 11$ .243

$\S 11(2)$ .243

$\S 77$. .244

$\S 79(2)(b)$ 245

§ 79(3) .245

$\S 80$. .244

$\S 82(1)(a)$ .245

$\S 82(2)$ .245

$\S 87(2)$ 239

$\S 90(3)$ 244

$\S 94$.

245

\section{United States}

Constitution . .54

Art. I, § 8 $.54,230$

Bayh-Dole Act (University and Small Business Procedures Act), Pub. L. No. 96-517, 94 Stat. 3015 (1980) (codified as amended at 35 U.S.C. $\S \S 200-212$ (2012)...46, 47, 53, 55, $57-62,67,68$

$\S 201(b)$ . .56

$\S 201(\mathrm{e})$ . .56

$\S 202$ .56

Berne Convention Implementation Act of 1988, Pub. L. No. 100-568, 102

Stat. 2853

$\S 3$ (b) .235

Computer Fraud and Abuse Act, 18 U.S.C. $\$ 1030$ (2008) 193

Conflict Minerals, 77 Fed. Reg. 56,274 (Sept. 12, 2012) (to be codified at 17 C.F.R. pts. 240 and 249b) ....106 Copyright Act, 17 U.S.C. $§ 101$ (1976). .229 $\S 101$ $226,229,231$

$\S 201$ .229

$\S 201(b)$ 229,231

$\S 302(a)$ .231

$\S 302(\mathrm{c})$. .231 $\S 410(c)$. .251
Copyright Act, 15 U.S.C. $\S \S 1$ et seq. (2012) $15,30,31$

$\S 106$ 30

$\S 501(a)$ 30

Cyber Economic Espionage

Accountability Act

(CEEAA) 199

Defend Trade Secrets Act, S. 2267, 113th Cong. (2014). .198

Economic Espionage Act (EEA) of 1996, 18 U.S.C. $\$ \$ 1831-837$ (2012)......193, 194, 195, 196, 198,

Fair Labor Standards Act 1938 (FLSA), Pub. L. No. 75-718, 52 Stat. 1060 (codified as amended at 29 U.S.C. § 201-219 (2012) .......7, 126-30, $131,133,134,135,136,137-40$, $141,142,143$

$\S 202(a)-(b)$ 129 $\S 203(\mathrm{~g})$ 127 $\S 213(\mathrm{a})(1)$ 126 $\S \S 206,207$ 127

Family and Medical Leave Act (FMLA), 29 U.S.C. § 2612(a)(1) (2012). 139

Foreign and Economic Espionage Penalty Enhancement Act of 2012, Pub. L. No. 112-269, 126 Stat. 2442 (2013)..........194, 195-6, 213

Health Care and Education

Reconciliation Act of 2010, Pub. L. No. 111-152, 124 Stat. 1029 (codified as amended in scattered sections of 42 U.S.C.) .................50

Health Insurance Portability and Accountability Act (HIPAA) 1996, Pub. L. No. 104-191, 110 Stat. 1936 (1996) (codified in scattered sections of 26 U.S.C. 29

U.S.C. \& 42 U.S.C.) . .49

Lanham Act § 45, 15 U.S.C. § 1127 (2006). $98,99,100,103$ $\S 1064, \S 1054$ .98

Leahy-Smith America Invents Act (AIA), Pub. L. No. 112-29, 125 Stat. 284 (2011) (codified at 35 U.S.C. \$ 1-375). 47,65 
xviii Managing the legal nexus between intellectual property and employees

$\S 102$

$\S 123$

Medicare Act (Social Security

Amendments of 1965, Pub. L. No. 89-97, § 102, 79 Stat. 286, 291332 (1965) (current version at 42 U.S.C. $\S \S 1395-1395 y y$ (2012)) $.48,49$

Medicaid Act (Social Security Amendments of 1965, Pub. L. No. 89-97, § 121, 79 Stat. 286, 343-52 (1965) (current version at 42 U.S.C. $\S \S 1396-1396 v$ (2012) 48,49

National Industrial Recovery Act (NIRA). .101

Patent Act. 35 U.S.C. $\S \S 1$ et seq. (2012).

$\S 101$ . .15

$\S 103$ .256

$\S 271(\mathrm{a})$

\& 271(b) $20,23,24,25,26,28$ $\S 271(\mathrm{c})$ $20,25,28,41$

Patient Protection and Affordable Care Act (ACA), Pub. L. No. 111-148, 124 Stat. 119 (2010) (codified as amended in scattered sections of the U.S.C.)..........47, 48, 50, 52, 53

Portal-to-Portal Act, 29 U.S.C. $\S \S 251-262$ (2012) 129

$\S 251(\mathrm{a})$. 129

$\S 254$ 129

Sherman Antitrust Act, 15 U.S.C §§ 1-7 $\S 1$ 263

Tariff Act of 1930 151

\$ 1337 (Section 337) .........8, 151-78, 196,213

$\S 1332(\mathrm{a})-(\mathrm{c})$ 151

$\S 1337$ (a)(1)(A) ..........152, 165, 175

$\S 1337(a)(1)(B)-(E) \ldots \ldots \ldots \ldots \ldots \ldots \ldots . . .152$

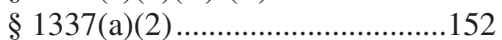

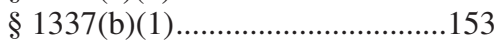

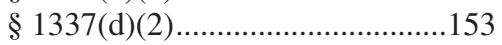

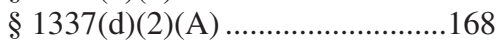

$\S 1337(d)(2)(B) \ldots \ldots \ldots \ldots \ldots \ldots \ldots \ldots . . .168$

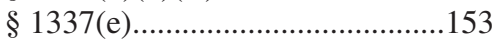

$\S 1337$ (f) ..............................153, 154

$\S 1337(f)(2) \ldots \ldots \ldots \ldots \ldots \ldots \ldots \ldots \ldots \ldots \ldots . . .163$
$\S 1337(\mathrm{~g})$ .54

$\S 1337(\mathrm{j})$ 153

Theft of Trade Secrets Clarification Act 2012, Pub. L. No. 112-236, 126

Stat. 1627 (2012)..194-5, 198, 213

Trade Act 1974 215

Uniform Trade Secrets Act (UTSA)

(model law) 193, 204, 209

Visual Artist Rights Act of 1990, P.L. No. 101-650 (1990) (codified as amended in scattered sections of 17 U.S.C.). .235

California Business \& Professional Code 16000 (2013)....................82

California Labor Code $\S \S 2870-72$ (West 2011) .................64

$\S 33515(\mathrm{c})$...................................232

Delaware Code, Del. Code Ann. 19

$\S 805$ (West 2006) 64

Illinois Compiled Statutes, 765. Ill. Comp. Stat. Ann. 1060/2 (West 2001)

Kansas Statutes, Kan. Stat. Ann. § 44130 (West 2008) .64

Minnesota Statutes, Minn. Stat. Ann. $\S 181.78$ (West 2006) .64

Nevada Revised Statutes, Nev. Rev. Stat. Ann. § 600.500 (West 2000)

Nevada St. Ann. § 439A.175 (2012) ..89

North Carolina General Statutes, N.C. Gen. Stat. Ann. §§ 66-57.1, 6657.2 (West 2012) .64

Utah Code, Utah Code Ann. § 34-39-3(6) (West 2011). .64

Washington Revised Code, Wash. Rev. Code $\S \S 49.44 .140-150$ (West 2008) 64

\section{European Union}

Regulation 593/2008 of the European Parliament and of the Council of 17 June 2008 on the Law Applicable to Contractual Obligations (Rome 1), 2008 O.J. (L 177) 6 . 249 
Directive 1991/250/EEC on the legal protection of computer programs (Computer Program Directive) 1991 O.J. (L 122) 42 .237 240,243

Art. 2(3) 237-8

Directive 93/98/EEC harmonizing the term of protection of copyright and certain related rights .238

Directive 2001/29/EC of 22 May 2001, Harmonisation of Certain Aspects of Copyright and Related Rights in the Information Society, 2001 O.J. (L. 167) 10 . .237

\section{International Treaties, Conventions and other Instruments}

Berne Convention for the Protection of Literary and Artistic Works, Sept. 9,1886 , as revised at Paris on July 24, 1971 and amended in 1979, S. Treaty Doc. No. 99-27 (1986) 216, 228, 233-5, $236,249,250$

Art. 5 .233

Art. 6bis ........234, 235, 239, 241, 244 Art. 6bis(1)....................................234 Art. 14bis(2).................................234 Art. 15(2) .234

Doha Declaration on TRIPS and Public Health 2001 .268
ILO Convention 138 (Minimum age for work) . .115 ILO Convention 182 (Eradication of the worst forms of child labor) ......115

Paris Convention ........................99, 216

Rome Convention .............................216

TRIPS Agreement: Agreement on Trade-Related Aspects of Intellectual Property Rights, Annex 1C of the Marrakesh Agreement Establishing the World Trade Organization, Apr. 15, 1994, 1867 U.N.T.S. 154 175,190 , 203, 215, 216, 217, 228, 233, 235-6, 249, 276 Art. 9(1) ........................................235

Art. 10 ..........................................216

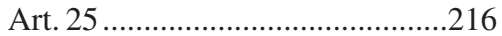

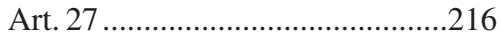

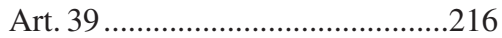

Art. 39(2) ....................................210

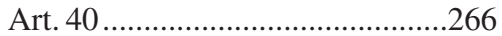

Art. 40(2) ....................................267

Trans-Atlantic Trade and Investment Partnership (T-TIP).........201, 218

Trans-Pacific Partnership Agreement...............................218

North American Free Trade Agreements (NAFTA) .234

United Nations Conference on Trade and Development (UNCTAD) Code of conduct related to international technology transfer agreements ..... 266-7 
Lynda J. Oswald and Marisa Anne Pagnattaro - 9781783479269 Downloaded from PubFactory at 04/26/2023 05:14:47AM 